\title{
LES ORIGINES RHÉTORIQUES DE LA THÉORIE DES TEXTES ARGUMENTATIFS
}

\begin{abstract}
A bstract. Lipińska Magdalena, Les origines rhétoriques de la théorie des textes argumentatifs [The Rhetoric genesis of the theory of argumentative texts], Studia Romanica Posnaniensia, Adam Mickiewicz University Press, Poznań, vol. XXVII: 2001, pp. 93-102. ISBN 83-232-1039-X, ISSN 0137-2475.
\end{abstract}

The present paper has been written for students whose intention is to learn the basic skills of composing argumentative texts such as essays, B.A. papers and M.A. theses. As it is widely known, the structure of all persuasive forms of writing adheres to specific norms and rules, which were clearly prescribed back in antiquity and which are mostly followed in contemporary textbooks on argumentative texts. In my paper, the current French theory of the latter has been explained through description of its classical roots. The analysis focuses in particular on the stages of argumentative composition, its inherent components and the typology of argumentative texts proposed by ancient Greeks and Romans.

«Poeta nascitur, orator fit» - «On naît poète, on devient orateur». Cette épigraphe des Anciens nous transmet leur message réconfortant d'après lequel pour bien parler et écrire le talent n'est pas tellement important. Ce qu'il faut, c'est un savoir théorique accompagné du travail et des exercices.

Si la théorie rhétorique est décrite d'une façon détaillée ainsi que celle des énoncés persuasifs contemporains, la dépendance qui existe entre l'une et l'autre n'est pas soulignée d'habitude dans les manuels actuels des textes argumentatifs. Nous comprenons par un texte argumentatif - un énoncé qui veut démontrer une thèse ou aboutir à une conclusion et pour cela il s'appuie sur un certain nombre d'arguments.

Or, il n'est pas possible; d'après nous, de comprendre les règles des exposés visant à convaincre sans se rendre compte de ce savoir classique qui constitue leur origine et leur fondement.

Si l'on réléchit sur la rhétorique aujourd'hui, il faut constater que c'est injuste que cet art de bien dire qu'on peut définir après Jerzy Ziomek comme la théorie de l'énoncé supérieur de la phrase, ne possède à présent que des connotations 
négatives. On les retrouve dans des expressions et des mots tels que: «c'est de la rhétorique», «lieux communs» ${ }^{1}$, sophisme. Toutefois la rhétorique est indispensable dans plusieurs domaines de la vie: diplomatie, publicité, vie politique, discours judiciaires, médecine ${ }^{2}$ et dans l'enseignement actuel ainsi que futur. Nous pensons ici au projet de l'introduction de la rhétorique en tant qu'une des matières à l'école, et à la théorie des textes argumentatifs nécessaire pour la préparation des travaux de licence, de maîtrise, des compositions. On ressent la nécessité de posséder ce savoir même dans la vie quotidienne. Savoir convaincre qui que ce soit de quoi que ce soit (ce qui était la devise des sophistes, philosophes qui ont contribué à la naissance de la rhétorique) n'est-ce pas une panacée dont on a besoin pour réussir sa vie? Les prémices énumérées ci-dessus nous ont persuadée de la nécessité de traiter le problème des origines rhétoriques de la théorie des textes argumentatifs.

Alors, comment écrire un bon discours destiné à convaincre? Répétons que ce sont les Anciens qui nous ont laissé la réponse à cette question en précisant aussi bien la méthode du travail que la distinction des parties constantes de chaque énoncé persuasif et une typologie des discours.

Avant de passer à la description succincte de ces points essentiels qui seront toujours confrontés à la théorie contemporaine des textes argumentatifs, nous présenterons quelques informations rudimentaires sur les représentants de la rhétorique antique, le caractère naturel de ses règles, les buts de chaque énoncé persuasif et les règles générales concernant le discours.

La rhétorique grecque est fixée à partir d'Aristote. Les rhéteurs latins et hellénistiques tels que Cicéron et Quintilien ont enrichi le système sans le modifier. Toutes les règles se rapportant aux textes argumentatifs n'ont pas été inventées d'une façon artificielle ni imposées autoritairement a priori mais font partie de la rhétorique dite naturelle ou descriptive, c'est-à-dire née de l'observation des discours prononcés dans la Grèce antique. Elles constituent donc la codification de ce qui a été d'abord appliqué pratiquement et d'une manière naturelle.

Presque chaque manuel des énoncés persuasifs précise leur but: «Plaire et convaincre». D'où vient cette formule? D'après les Anciens, le discours devrait influencer: la raison, la volonté et les sentiments. Cette tripartition correspond à trois éléments de l'âme humaine d'après Platon: la raison, la volonté et la passion. Il faut donc informer, émouvoir et plaire. La fonction la plus importante est celle qui tend à informer (on l'appelle - informative-enseignante). Autrement dit, chaque texte conçu pour convaincre doit être avant tout clair et logique.

La construction du texte argumentatif est subordonnée à deux importantes règles générales: la règle du caractère organique du discours et la règle de son caractère fonctionnel. D'après la première, formulée par Platon, chaque énoncé

${ }^{1}$ Le sens actuel de cette expression - «banalités» a comme origine la signification "des arguments, développements et preuves applicables $\dot{r}$ tous les sujets», v. P. Robert, J. Rey-Debove, A. Rey, Nouveau Petit Robert, Dictionnaires Le Robert - Paris 1993, p. 1283.

${ }^{2}$ Il s'agit surtout de la pithiatrie qui est un traitement par la persuasion accompagnée de la suggestion. 
persuasif, comme un organisme vivant, pour bien fonctionner a besoin de tous ses membres, de toutes ses parties et par conséquent, il doit se caractériser par la cohésion, l'ordre, la disposition correcte des éléments. La deuxième souligne le fait que l'organisation du discours doit être conforme à son but qui indique quels mots et pensées doivent être mis en relief. L'application de cette règle est essentielle dans l'étape de l'élaboration du plan en décidant de l'harmonie et des proportions des éléments de l'énoncé.

Comme nous l'avons déjà mentionné le système de la rhétorique antique précise les étapes du travail (connues sous le nom de parties de la rhétorique) ayant pour but l'élaboration du discours. Elles sont les suivantes:

1) l'invention - dans laquelle il est question de comprendre le sujet et de rassembler toutes les idées (tous les arguments);

2) la disposition - qui consiste à mettre en ordre ces idées, à faire un plan. C'est ici qu'on distingue les parties constantes de chaque énoncé persuasif: l'exhorde, la narration, la confirmation et la péroraison;

3) l'élocution - dans laquelle il s'agit de rédiger un discours en lui donnant un style. On y précise les types de figures stylistiques;

4) la mémoire - c'est-à-dire l'apprentissage par coeur d'un texte écrit;

5) l'action - dans laquelle on s'exerce à le prononcer (l'étude des gestes, de la mimique, de la maîtrise du souffle, de la diction).

Comme les deux demières se rapportent aux textes destinés à être prononcés, on les omettra dans nos réflexions.

Dans l'étape de l'invention, le rassemblement des données s'effectue à la base de la mémoire mais aussi des livres (p.ex. des recueils de proverbes ou de citations). Ces seconds constituent ce que les Anciens appelaient topoi (lieux) - des sources dont l'orateur puisait des arguments. Les idées recherchées doivent présenter un problème traité comme digne d'attention. Il faut aussi qu'elles aboutissent à la persuasion. Dans la Grèce antique, on a élaboré également une méthode pratique pour trouver des idées, à savoir - une grille de questions qu'il faut se poser: Qui? Quoi? Où? Au moyen de quoi? A l'aide de qui? Pourquoi? De quelle façon? Quand?

La phase de l'invention correspond aux points suivants précisés par un manuel contemporain des textes argumentatifs ${ }^{3}$ :

1. Le choix du sujet. On conseille de le faire assez rapidement et de ne pas revenir sur un premier choix après quelques heures du travail (dans les conditions d'un examen). Si le sujet est unique et paraît difficile il faut patienter, se concentrer sur lui, même assez longtemps.

2. L'étude approfondie du sujet. Il est nécessaire de lire le sujet plusieurs fois afin de le comprendre à fond. Il faut essayer de cemer le problème et de l'énoncer avec ses propres mots.

3. La recherche des idées. On recommande de noter en vrac toutes les idées qui

${ }^{3}$ P. Dés almand, P. Tort, Du plan à la dissertation, Hatier, Paris, p. 60-61. 
viennent à l'esprit (aussi bien les exemples que les illustrations littéraires, les citations, etc.). Pour s'aider on peut rattacher le sujet à une expérience personnelle.

La disposition équivaut à l'établissement du plan ${ }^{4}$. Ce qui est à souligner dans la théorie actuelle, c'est que l'étape de l'élaboration du plan doit être suivie de la rédaction des grandes lignes de la conclusion. Ce n'est qu'après qu'on conseille d'écrire l'introduction et de rédiger tout le reste du travail. Nous retrouvons le même ordre dans la théorie classique ( $v$. les informations sur l'exhorde). Le type de plan doit être adapté au sujet du travail (conformément à la règle du caractère fonctionnel du discours). Les manuels contemporains des textes argumentatifs distinguent entre autres deux genres principaux de plans: le plan dialectique et le plan "par le triple point de vue». Le premier se compose de trois optiques: 1) la thèse qui constitue une défense d'un certain point de vue sur la question; 2) l'antithèse qui caractérise le point de vue contraire; 3) la synthèse, c'est-à-dire l'établissement d'une vérité moyenne. Le deuxième plan possède également une construction tripartite: 1) la présentation du point de vue de l'auteur de la citation constituant le sujet du travail; 2) la description du point de vue habituel sur la question; 3) la caractéristique du point de vue de l'auteur du travail. Il faut veiller à ce que ça ne soit pas une simple juxtaposition des points de vue mais leur confrontation continuelle.

On peut observer que ces deux types de plans suivent l'une des règles de la disposition précisée par les Anciens d'après laquelle les voies d'analyse ne peuvent pas être trop nombreuses. On en accepte trois tout au plus.

C'est aussi dans cette étape de l'élaboration du texte argumentatif qu'il faudrait placer la théorie classique des transitions qu'on appelle aujourd'hui «procédés, techniques d'enchaînement». Toutes les parties du discours ainsi que des arguments doivent être donc enchaînés au moyen de mots ou de locutions qui les mettent en relief, qui soulignent la logique et l'organisation de l'énoncé.

Les Anciens conseillent de même de ne pas avoir peur de répéter, sous des formes différentes, des idées, des arguments les plus importants. Cette reprise appelée «parallélisme rhétorique» est très utile car elle renforce le pouvoir persuasif du discours.

La notion de style est essentielle à la phase de l'élocution. Nous n'allons pas nous attarder sur le schéma des styles anciens connu sous le nom de «roue de Virgile» où l'on précisait, p.ex. des prénoms des héros, leurs attributs et le décor typiques de chaun de trois styles. Il n'est pas pourtant sans intérêt de citer ces derniers et de les caractériser d'une façon générale pour enfin rappeler des remarques classiques d'ordre normatif, toujours actuelles et négligées dans les manuels contemporains des textes argumentatifs.

L'Antiquité distinguait le style noble, simple et tempéré. Le premier (grave ou sublime) devait être employé dans les sujets importants, grandioses. Il s'exprimait

${ }^{4}$ La théorie concernant les èlements constants de chaque discours tels que l'exhorde, la narration, etc. sera traitée d'une façon détaillée aprěs la description des parties de la rhétorique. 
par les moyens propres à susciter les sentiments, surtout par les figures rhétoriques. Le style simple (tenu) sobre et précis s'appliquait le mieux au but didactique. Privé de tropes, il était communicatif, marqué par l'économie, la clarté et la modestie. Le style tempéré (medium) passait pour un juste milieu quant à l'emploi des figures en exigeant beaucoup de mesure. C'était un idéal de l'art de bien parler d'après les théoriciens de la rhétorique. Il faisait place à l'anecdote et à l'humour.

Écrire ou parler bien cela veut dire surtout, savoir adopter chaque fois le style qui convient le mieux à la situation (le noble - pour émouvoir, le simple - pour instruire, le tempéré - pour plaire). Mais à part la conformité avec la situation en quoi consiste le bon style? Il devrait avoir trois qualités: correction, clarté et ornementation. La correction, c'est-à-dire l'absence de fautes, l'emploi des termes précis, non équivoques, non généraux. La clarté consiste dans l'application des notions justes, l'ordre correct de la phrase. C'est elle qui fait éviter des mots abstraits, étrangers ou empruntés. L'ornementation, c'est autrement dit le lexique riche, la présence de l'humour, la variété des moyens rhétoriques, l'usage contrôlé des figures stylistiques telles que: la répétition, la comparaison, l'antithèse, la métaphore, la périphrase, l'hyperbole, l'anaphore, la question rhétorique, l'apostrophe, etc. (pour ne citer que celles qui sont les plus connues).

Après avoir décrit la méthode pour rédiger les textes argumentatifs nous traiterons leurs éléments constants en présentant d'abord la théorie rhétorique et ensuite les conceptions contemporaines qui en découlent. Voilà les parties de chaque discours distinguées par les Anciens (à gauche) lesquelles correspondent aux points principaux du schéma général d'une dissertation. Cette dernière est un exemple typique d'un texte argumentatif.

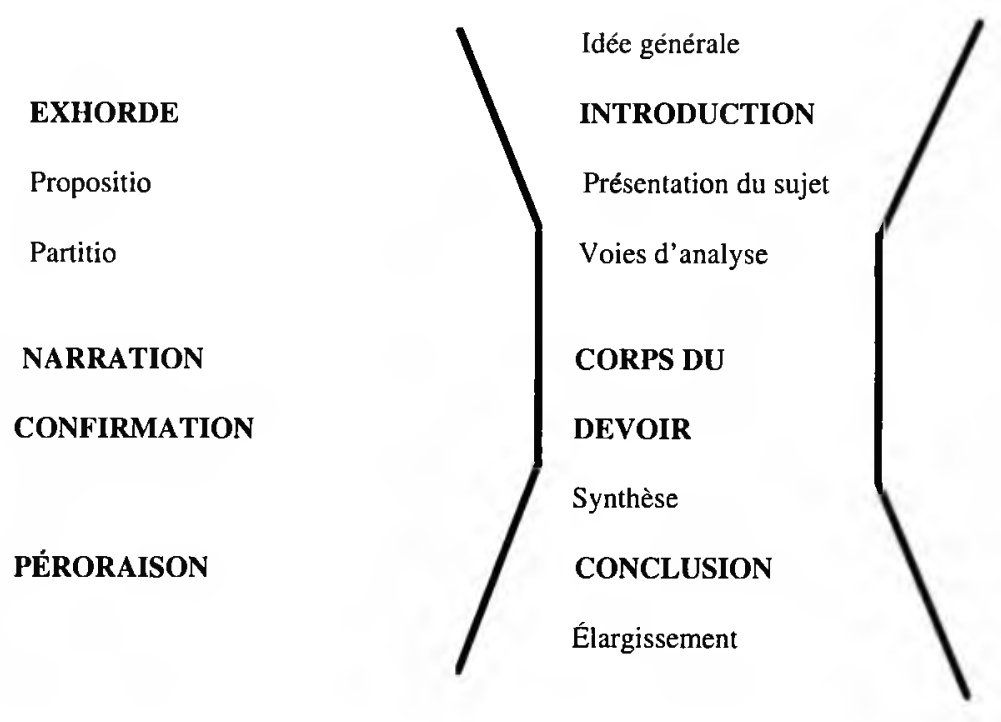


L'exhorde est la première et la plus importante partie du discours car ce qui décide surtout de l'efficacité de la persuasion, c'est une impression initiale. Son but est de «rendre l'auditoire attentif, bienveillant et docile». Il doit annoncer le sujet mais sa fonction principale est de plaire. Il faut aussi qu'il montre l'importance de la question traitée. On y aboutit: 1) en portant un jugement de valeur sur le sujet; 2) en mettant en relief la nécessité de le traiter; 3) en accentuant son caractère intéressant, attrayant.

L'exhorde doit être adapté aux circonstances du temps, du lieu, des événements, des personnes. Pour plaire, son originalité du point de vue du contenu et de la forme stylistique est nécessaire (p.ex. le caractère imagé). On exige enfin qu'il soit concis, c'est-à-dire proportionnel par rapport aux autres parties du discours. On le juge inconvenable quand il est général (quand il se prête à plusieurs sujets), neutre du point de vue émotionnel ou non lié au problème traité.

Les Anciens précisaient deux éléments importants de cette partie de la disposition, à savoir: le propositio et le partitio. Le premier placé vers la fin de l'exhorde constitue le point de départ du discours en posant un problême. C'est une phrase simple, affirmative, négative ou interrogative, non équivoque. Le deuxième est un développement du propositio qui explique celui-ci en citant ses parties constitutives. Il faut que le partitio (ou le plan général de l'énoncé) soit 1) simple et clair, autrement dit résultant naturellement du sujet; 2) pas trop développé (se composant de trois éléments au maximum). Si l'objet du discours paraît difficile à diviser il vaut mieux ne pas le faire; 3 ) exhaustif. Il faut qu'il concerne «tout le sujet et rien que le sujet». En traçant le partitio il faut veiller à ce que la partie théorique précède la partie pratique et tout ce qui est agréable pour le locuteur soit placé avant des éléments désagréables.

On peut observer que la formulation du partitio déjà dans l'exhorde présuppose un travail préalable (la recherche des arguments, l'établissement du plan) ce qui fait que ce premier fragment du discours devrait être rédigé en principe à la fin de la disposition.

La théorie moderne de l'introduction reprend les points essentiels de l'exhorde en divisant celle-ci en trois éléments: 1) l'entrée en matière; 2) la présentation du sujet (qui correspond au propositio des Anciens; 3 ) les voies d'analyse (ayant leur origine dans le partitio).

L'entrée en matière doit surtout intéresser le lecteur. Le plus simple est de commencer par une phrase affirmative à caractère général mais d'autres formes telles que: une question, un exemple court, une citation (différente de celle du sujet) suscitent chez le lecteur, un plus grand intérêt.

Présenter le sujet signifie le décrire comme si le lecteur ne le connaissait pas. On peut le recopier en entier s'il est court ou, s'il est long, on extrait l'essentiel pour le préciser en deux ou trois lignes. Il faut se garder de ne pas transformer le sujet et de ne pas le traiter. On déconseille également de donner la réponse au problème ou de présenter son opinion ce qui est le rôle de la conclusion. Si la formulation du 
thème du travail semble équivoque, il est indispensable de préciser dans quel sens on prend les mots-clés du sujet.

Tracer les voies d'analyse consiste à citer le plan suivi dans le développement. On peut le faire sous forme d'interrogations directes.

La question du caractère proportionnel de l'exhorde est également reprise par la théorie moderne d'après laquelle l'introduction devrait occuper le huitième du devoir (p. ex. $6-8$ lignes pour un travail de $2-3$ pages).

Entre l'exhorde et la péroraison il y a la narration et la confirmation lesquelles correspondent à ce qu'on appelle aujourd'hui «développement» ou «corps du devoir».

La narration est une explication de l'objet du discours, un exposé des faits. D'après Cicéron elle peut être «historique», c'est-à-dire présentant l'état de recherches sur un sujet traité, ou «philosophique» constituée par des définitions des mots-clés du sujet. Les traits caractéristiques de cette partie du discours découlent de sa fonction principale qui est celle d'instruire. Elle doit être claire, concise, authentique, autrement dit basée sur les faits liés logiquement et s'appuyant sur les sources authentiques.

Dans la confirmation, on cite les arguments et on réfute. Elle se compose donc de l'argumentation et de la réfutation. En décrivant l'argumentation on ne peut ne pas répondre aux questions suivantes: 1) Où peut-on trouver des arguments? 2) Comment devraient-ils être? 3) Quels sont leurs types? 4) Comment les disposer? 5) En quoi consiste le problème de l'éthos et du pathos?

Les arguments peuvent être puisés dans la définition d'un mot-clé, dans l'étymologie de celui-ci, dans l'énumération des éléments d'une notion importante pour le sujet, dans la comparaison, dans l'antithèse.

La méthode la plus simple pour construire l'argumentation consiste à trouver des preuves pour et contre et à choisir les plus convenables. Il faut se garder de prouver des choses évidentes. Les arguments devraient être vraisemblables mais pas forcément vrais.

Nous ne citerons que quatre exemples d'arguments applicables à plusieurs types de textes argumentatifs: 1) l'approbation de la cause et la négation des conséquences. Il se résume par la phrase: «C'est vrai en théorie, mais faux en pratique»; 2) argumentum ad auditorem où l'on s'adresse aus sentiments de l'auditeur, le plus souvent sous forme de question rhétorique; 3) argumentum ad hominem (adapté à l'adversaire) dans lequel on emploie les arguments d'un adversaire (réel ou imaginé), p.ex. quand quelqu'un défend le suicide on lui pose la question: «Pourquoi ne te suicides-tu pas?»; 4) argumentum ad verecundiam (se rapportant au respect ou à l'estime) où l'on cite l'opinion de quelqu'un qui jouit de l'autorité (d'un personnage illustre) ou bien une citation, un proverbe (c'est-à-dire une formule) où l'autorité de «il» est remplacée par celle de «on».

Il est obligatoire de disposer des arguments d'après un ordre. On distingue ordo Homericus (l'ordre Homérique) qui consiste à placer au début et à la fin des 
preuves fortes et au centre - les preuves faibles. On peut aussi ranger les arguments en gradation (des plus forts aux plus faibles ou inversement).

Afin de mieux convaincre il faut savoir émouvoir, plaire, séduire le locuteur. Pour y aboutir, le discours doit entre autres savoir transmettre des sentiments. C'est possible à condition que l'orateur les ressente lui-même. Ce qui s'impose donc, c'est l'étude de l'éthos et du pathos. L'éthos est le caractère que doit présenter l'orateur pour gagner son public. Il faut qu'il paraisse sensé, sincère et sympathique. L'efficacité de la persuasion dépend beaucoup de la confiance qu'il inspire. Le pathos, ce sont des passions servant à émouvoir et à vaincre les auditeurs. Les Anciens ont répertorié les fautes oratoires typiques liées à l'emploi des moyens émotionnels. Ce sont entre autres: le passage trop rapide de la description d'un sentiment fort au raisonnement logique (autrement dit, l'absence de pause psychologique) et la succession des sentiments contraires, p.ex. l'application d'une plaisanterie dans un discours sérieux.

Le point final de la confirmation est une digression - un récit, une méditation qui renforce la preuve par l'émotion. Mais la partie la plus convenable pour exprimer les sentiments, c'est la péroraison.

La réfutation des arguments est nécessaire quand les locuteurs sont prévenus contre une thèse défendue dans le discours. Dans ce cas-là, la partie de l'énoncé en question devrait précéder l'argumentation.

La plupart des remarques rhétoriques concernant la manière de rédiger la confirmation et la réfutation pourraient s'appliquer à la méthode d'écrire la partie essentielle d'un énoncé argumentatif, appelée corps $d u$ devoir. D'après les théoriciens contemporains, il faut que celui-ci soit basé sur un plan se caractérisant par: ordre, rigueur logique et progression. Nous avons déjà mentionné deux genres de plan: dialectique et celui par le triple point de vue. Paul Désalmand et Patrick Tort, dans leur ouvrage $\mathrm{Du}$ plan à la dissertation ${ }^{5}$, citent aussi: le plan problème-causes-solutions, le plan inventaire, le plan comparatif, le plan explication-illustration d'une formule et commentaire et le plan suggéré par le sujet. On peut observer que certains types énumérés ci-dessus correspondent aux sources d'arguments (topoi ou lieux), p.ex. le plan dialectique est fondé sur l'antithèse, le plan comparatif - sur la comparaison.

La péroraison est la derière partie du discours qui contient la synthèse du contenu de celui-ci et qui a le caractère émotionnel. On y attache une grande importance car c'est elle qui décide de l'efficacité de la persuasion. D'après Aristote, il faut qu'elle remplisse quatre rôles: plaire, élargir ou diminuer la thèse fondamentale, émouvoir et mettre en relief les arguments principaux. Il faut plaire pour gagner définitivement le public. Dans la mise en relief des arguments les plus importants, Aristote conseille de les répéter plusieurs fois pour qu'ils deviennent compréhensibles. D'après le mểme philosophe, la conclusion devrait se caractériser par le style non conjonctionnel ce qui permettrait de la différencier du reste du

\footnotetext{
${ }^{5}$ Op. cit.
} 
discours. Les théoriciens de la rhétorique veillaient surtout à la construction de la dernière phrase étant un équivalent de la pointe poétique ou du dernier accord dans la composition musicale.

Les manuels des textes argumentatifs contemporains redisent après les rhétoriciens toutes les fonctions de cette partie finale de l'énoncé, c'est-à-dire: 1) la synthèse du développement; 2) la présentation de l'opinion personnelle (ce qui correspond au caractère émotionnel souligné par les Anciens); 3) l'élargissement du débat (ceci équivaut dans la théorie classique à l'élargissement ou à la diminution de la thèse).

On rappelle que la synthèse ramassant l'essentiel du développement en quelques phrases devrait répondre au sujet posé en introduction. L'élargissement ou l'ouverture consiste à montrer comment le sujet n'est qu'un aspect d'un problème plus large. On conseille également de terminer le travail par une citation appropriée ce qui correspond à la pointe poétique tellement prisée par les codificateurs grecs et romains. Nous y retrouvons l'intention de plaire aux auditeurs.

La théorie contemporaine souligne aussi le caractère proportionnel de la conclusion par rapport au reste de l'énoncé. Il faut que celle-ci soit assez courte, p.ex. quatre à six lignes pour le devoir de deux, trois pages.

Les Anciens nous ont laissé également une typologie des discours argumentatifs correspondant aux sujets possibles avec les indices concernant: buts, critères et types d'argumentation, styles, temps propres à chacun d'eux.

\begin{tabular}{|l|l|l|l|l|l|}
\hline $\begin{array}{c}\text { Type de } \\
\text { discours }\end{array}$ & $\begin{array}{c}\text { But du } \\
\text { discours }\end{array}$ & $\begin{array}{c}\text { Critère dont } \\
\text { devrait se } \\
\text { servir } \\
\text { l'orateur en } \\
\text { composant le } \\
\text { discours }\end{array}$ & $\begin{array}{c}\text { Types } \\
\text { d'arguments } \\
\text { propres à chaque } \\
\text { discours }\end{array}$ & Styles & \multicolumn{1}{|c|}{$\begin{array}{c}\text { Temps } \\
\text { employé }\end{array}$} \\
\hline judiciaire & accuser ou & la justice & $\begin{array}{l}\text { le raisonnement } \\
\text { (déduction ou } \\
\text { induction) }\end{array}$ & simple & passé \\
\hline délibératif & conseiller & l'utilité & $\begin{array}{l}\text { l'exemple (les } \\
\text { précédents }{ }^{6} \text { ) }\end{array}$ & sublime & futur \\
\hline $\begin{array}{l}\text { épidictique } \\
\text { (ou } \\
\text { démonstratif) }\end{array}$ & $\begin{array}{l}\text { l'éloge ou le } \\
\text { blâme }\end{array}$ & $\begin{array}{l}\text { l'esthétique et } \\
\text { la morale }\end{array}$ & $\begin{array}{l}\text { l'amplification' } \\
\text { (le développe- } \\
\text { ment) }\end{array}$ & tempéré & neutre (plutôt \\
le présent)
\end{tabular}

${ }^{6}$ Le précédent est un cas particulier d'exemple qu'on peut résumer de la façon suivante: «Si X a pu faire ou pu recevoir ceci, pourquoi pas Y\%. Avant de s'en servir, il faut s'assurer que la relation est la męme dans les deux cas. Voilr un specimen d'un emploi incorrect d'un précèdent: «Un pčre reproche r' son fils d'ętre l'amant de sa grand-mčre. Mais, répond le fils, vous couchez avec ma mčre, pourquoi ne coucherais-je pas avec la vôtre?n, v. O. Reboul, La rhétorique, P.U.F., 1984.

${ }^{7} \mathrm{~L}$ 'amplification (ou l'enrichissement) consiste dans l'emploi des figures telles que: l'hyperbole, la répétition, la métaphore. Son but est de démontrer qu'une chose est petite ou grande, bonne ou mauvaise, juste ou injuste. Ses principales méthodes sont les suivantes: l'amplification par la gradation (p.ex. l'hyperbole), par la comparaison (de deux exemples: un exemple important et un exemple faible), par l'accumulation (p.ex. des arguments concernant le męme objet). 
Nous venons de démontrer que les principes contemporains d'après lesquels on construit les textes argumentatifs ont leur origine dans la théorie rhétorique qui précise aussi bien les étapes de la création de ceux-ci (ou les parties de la rhétorique: l'invention, la disposition, l'élocution, la mémoire, l'action) que leurs élếments constants (l'exhorde, la narration, la confirmation, la péroraison). Elle caractérise aussi les types de discours (judiciaire, délibératif et épidictique) en fournissant les informations portant sur les buts, les critères et les types d'argumentation, les styles ainsi que les temps propres à chacun d'eux.

Les thèses principales de la rhétorique dont les grandes lignes ont été à peine esquissées dans le présent travail constituent une source toujours inépuisable fondant la base non seulement de l'art d'écrire des énoncés persuasifs mais aussi des sciences telles que: la logique, la psychologie, l'éthique, la sociologie, la philosophie, la grammaire, la théorie de la littérature, la stylistique, la poétique historique. En y réfléchissant il est difficile de ne pas donner raison au proverbe français: «Paroles vieillies, paroles sages».

\section{BIBLIOGRAPHIE}

B raud M. (1991), L'expression écrite et la composition française, C \& D International, Kraków.

Dés al mand P. (1977), Tort P., Du plan à la dissertation, Hatier, Paris.

Gardes-Ta mine J. (1966), La rhétorique, Armand Colin/Masson, Paris.

Korolko M. (1998), Sztuka retoryki, Przewodnik encyklopedyczny, Wiedza Powszechna, Varsovie.

Reboul O. (1984), La rhétorique, P.U.F.

Robert P., Rey-Debove J., Rey A. (1993), Le nouveau Petit Robert, Dictionnaires Le Robert, Paris. 